\title{
Comparative Study of Outcome of two Methods (Sutures Vs Staples) of Skin Closure in Orthopedic Surgery
}

\author{
Gohiya A $\mathbf{A}^{1}$, Gupta DC ${ }^{2}$, Gaur $\mathbf{S}^{3}$ \\ ${ }^{1}$ Dr Ashish Gohiya, Associate Professor Department of Orthopedic, ${ }^{2}$ Dr Dinesh Chandra Gupta, ${ }^{3}$ Prof Sanjiv Gaur, \\ Head, Department of Orthopaedic. All are affiliated with Gandhi Medical College, Bhopal, MP, India
}

Address for Correspondence: Dr Ashish Gohiya, Email: gohiyapoorva@gmail.com

\begin{abstract}
Introduction: Surgical site wound closure plays vital role in post-operative success. The use of either sutures or staples for skin approximation is a frequently debated issue. We compared the outcome of using staples versus sutures in elective orthopedic surgical procedures. Material and Methods: Study was conducted in tertiary care teaching hospital in central India over a period of 2 years which included all patients aged between 18 to 80 years undergoing elective open surgeries. Computer generated random number randomized them in two groups. Skin closure was done by either monofilament nylon or metallic staples. The wounds were assessed for infection, wound dehiscence, pain during removal on the basis of VAS pain scale. Follow up was done in the 6th week in terms of patient satisfaction using visual analogue scale (VAS) and validated cosmesis scoring system Hollander Wound Evaluation Score (HWES). Results : Total 503 wounds were included in this study (262 in suture and 241 in staple groups), 14 (5.3\%) wounds were culture positive in the suture group within 8th postoperative day as compared to 36(14.9\%) in staples group with $\chi 29.65$ and ( $\mathrm{p}=0.002)$ which is significant. 320 wounds with $>10 \mathrm{cms}$ incision were analyzed (166 in suture and 154 in staple group) out of which 12 (7.22\%) were infected in suture group and $24(15.5 \%)$ in staple group with $\chi 2$ was 7.6 and $(\mathrm{p}=0.006)$ that statistically significant. Conclusion: We found a statistically significant higher incidence of superficial wound infection following skin closure by metallic staples as compared to monofilament nylon.
\end{abstract}

Key word: Nylon, Staples, Infection, kin closure, Post operative infection

\section{Introduction}

For over 50,000 years physicians have been searching for ideal suture material [1]. Surgical site wound closure plays a vital role in post-operative success. This effect is magnified with regards to commonly performed elective orthopedics procedures. The use of either sutures or staples for skin approximation remains a contested subject as this may have a significant impact on both patient safety and surgical outcome.

The type of suture material for skin closure is also reported to influence postoperative wound complications. Following orthopedic procedures wound complications are one of the main sources of illness. Orthopedic surgeons use both metallic staples and sutures to close wounds. Staples are regarded as quicker as and easier than sutures, but staples are more likely to cause infection and may also be more expensive [2].

Manuscript received: $1^{\text {st }}$ Nov 2013

Reviewed: $16^{\text {th }}$ Nov Aug

Author Corrected; $9^{\text {th }}$ Nov 2013

Accepted for Publication: $15^{\text {th }}$ Dec 2014

International Journal of Medical Research and Review
Slade Shantz JA et.al have shown the mechanical equivalence of stapled and sutured wounds. They also have found that no comparative trial exists that has the statistical power to determine if the rate of infection in wounds closed with staples is different from that in wounds closed with sutures in orthopaedic procedures [3].

\section{Aims \& Objectives}

-To compare clinical outcome of skin closure by monofilament nylon and metallic staples in orthopedic surgeries.

-To evaluate outcome of wound after skin closure by above two methods by using Hollander wound evaluation score (HWES) [4] and Visual analogue patient satisfaction scale (VAS).[5]

\section{Materials \& Methods}

Study design- Prospective randomized controlled study. Material- Patients admitted at Department of 
Research Article

Orthopedics (Hamidia Hospital,) GMC Bhopal with close fractures and for any other open elective surgeries were included in the this study. Informed consent was taken from each patient and each patient was randomized and a random number given to each patient as per computer randomization method to include in two study groups (Nylon and Staple group). Each patient was screened in as per inclusion and exclusion criteria and patient data was collected on case report forms, which were later entered into an electronic database (MS Excel, Microsoft).

\section{Inclusion criteria}

1. Age $>18$ years and $<80$ years

2. All elective open orthopedic surgeries

3. Incision size $>5 \mathrm{cms}$

4. Healthy patients with no co-morbidities

5. $\mathrm{HB}>\operatorname{logm} \%$

\section{Exclusion criteria}

1. Open fractures

2. Active infections (any site)

3. Immunocompromised patients

4. Foot surgery (any site)

5. Hand surgery (any site)

6. Minimal invasive surgeries (closed nailing, pining, percutaneous screw fixation, Arthroscopic procedures

\section{Methods}

Patients who fulfilled inclusion criteria and who gave consent for the study were enrolled in this study. Standard preoperative preparations were followed for both the study groups. For limb surgery where tourniquet required, tourniquet was inflated after limb elevation for 4 to 5 minutes and remained inflated till closure was completed. Length of incision (divided into two groups $<10 \mathrm{cms}$ and $>10 \mathrm{cms}$ ) was noted. Drain was put in all hip and knee surgeries. At the completion of surgery closure of wound done in layers by absorbable sutures till subcutaneous layer and skin was closed by either monofilament nylon or metallic staples as per randomization. Duration of skin closure was noted. Each patient was given same intravenous antibiotic till 5 th postoperative day. Each wound was assessed at $2 \mathrm{nd}$, 5 th and 8 th postoperative days. Suture/staple removal done between 11 th and 14th post-op days. Any discharge from wound during wound inspection was swabbed and sent for pus culture and sensitivity and if reported growth then it was considered as infection. Wound dehiscence was noted. Requirement of secondary procedures like wound debridement, wound wash and resuturing was noted. And pain occurring during removal was also evaluated on VAS pain scale. Follow up was done at 6 th week in terms of cosmesis by using a validated cosmesis scoring system, Hollander wound evaluation score (HWES)[4] and patient satisfaction using Visual Analogue Score (VAS) system[5]. A blinded statistician using chi square test did statistical analysis of observations.

\section{Observation \& Results}

A total of 513 patients were enrolled in this present study. 2 patients died postoperatively and 8 patients lost to follow up so effective number of patients included in this final study was 503 out of which 262 were in nylon group ( $n=262)$ and 241 in staple group $(n=241)$.

The mean age group was 41.6 years. 348 (69.1\%) were male and 155 (30.8\%) were female.

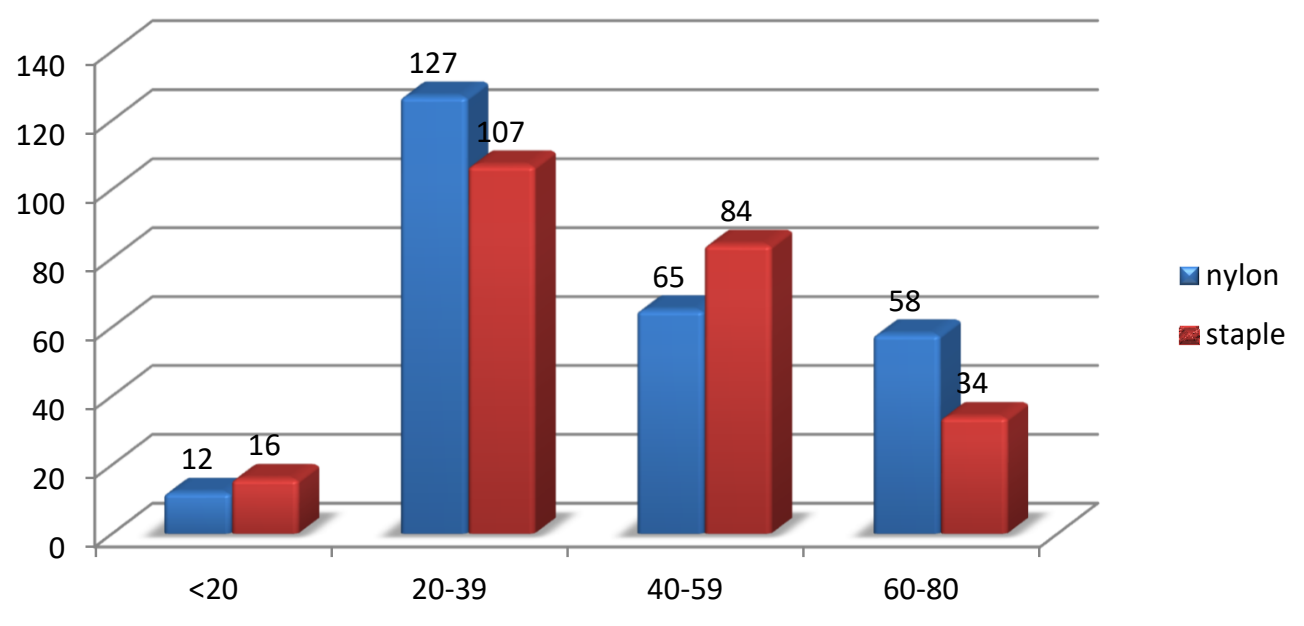

Anatomical Distribution of Surgeries 
Hip-192, Knee and around knee-98, ankle-98, Upper limb-115

A total of 183 wounds had incision size $<10 \mathrm{cms}$ (96 in nylon group and 87 in staples group) whereas 320 wounds had $>10 \mathrm{cms}$ incision (166 in nylon group and 154 in staple group).

\section{Duration of Skin Closure}

The mean duration of closure in nylon group was 4.24 minutes and in staple group was 0.96 minutes.

\section{Post Op Discharges From Wounds}

Wound discharges in hip surgery were maximum at first check dress 65 (75.5\%) in nylon group and 71 (75.5\%) in staple group $(\mathrm{p}=0.16)$. Comparing two methods of closure in knee surgery wound discharge at first check dress was statistically insignificant $(p=0.23)$ but in ankle and upper limb surgeries it was statistically significant with $(p=0.04)$ and $(p=0.01)$ respectively.

\section{Infection Rate}

$14(5.3 \%)$ wounds were culture positive within 8th postoperative day in nylon group as compare to $36(14.9 \%)$ wounds in staples group with $\chi 29.65$ and $(\mathrm{p}=0.002)$ that was quite significant.

In hip surgeries 5(5.1\%) wound infection were recorded in nylon group, $13(13.8 \%)$ in staple group with $\chi 24.3$ and $(p=0.038)$ which is statistically significant. In knee and around the knee surgeries- $3(6 \%)$ wound infection were in nylon group whereas $8(16.6 \%)$ infection in staple group with $\chi 22.8$ and $(\mathrm{p}=0.09)$. In ankle surgeries-4 (7.6\%) wound infections were in nylon group as compared to $7(21.7 \%)$ infection in staple group with $\chi 23.93$ and ( $\mathrm{p}=0.04)$ which was statistically significant. In upper limb surgeries only $2(3.2 \%)$ wound infections were found in nylon group and $5(9.4 \%)$ infection in staple group with $\chi^{2} 1.93$ and $(\mathrm{p}=0.16)$

Table 1: Infection rate in diferent anatomical site of surgery

\begin{tabular}{|l|l|l|l|l|}
\hline Group name & $\begin{array}{l}\text { No of infection in } \\
\text { hip surgery(\%age) }\end{array}$ & $\begin{array}{l}\text { No of infection knee } \\
\text { and around knee } \\
\text { surgery(\%age) }\end{array}$ & $\begin{array}{l}\text { No of infection } \\
\text { ankle surgery } \\
\text { (\%age) }\end{array}$ & $\begin{array}{l}\text { No of infection } \\
\text { upper limb } \\
\text { surgery (\%age) }\end{array}$ \\
\hline Nylon group $(\mathrm{n}=262$ & $9(3.4 \%)$ & $4(1.5 \%)$ & $5(1.9 \%)$ & $7(2.6 \%)$ \\
\hline Staple group $(\mathrm{n}=241)$ & $11(4.5 \%)$ & $5(2 \%)$ & $6(2.4 \%)$ & $7(2.9 \%)$ \\
\hline
\end{tabular}

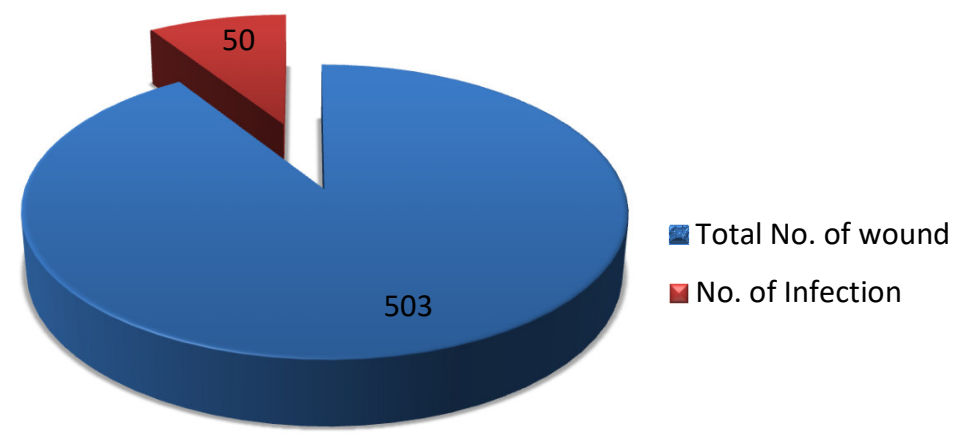

\section{Infection Rate in of Wounds of different sizes}

4 wounds with size $<10 \mathrm{cms}$ were infected in nylon group and $12(13.7 \%)$ in staples group with $(\mathrm{p}=0.02)$.

12 wounds with size $>10 \mathrm{cms}$ were found to be infected in nylon group whereas $24(15.5 \%)$ wounds were found in staple group with $\chi 27.6$ and $(\mathrm{p}=0.006)$. 
Table 2: Infection rate in wound size $>10 \mathrm{~cm}$

\begin{tabular}{|l|l|l|}
\hline Group & Total no wounds & No of infection(\%age) \\
\hline Nylon group & 166 & $12(7.22 \%)$ \\
\hline Staple group & 154 & $24(15.5 \%)$ \\
\hline
\end{tabular}

\section{Wound Dehiscence}

$25(9.2 \%)$ in nylon group $(n=262)$ and $29(12.4 \%)$ in staple group $(n=241)$ with $(\mathrm{P}=0.1)$ which is not statistically significant.

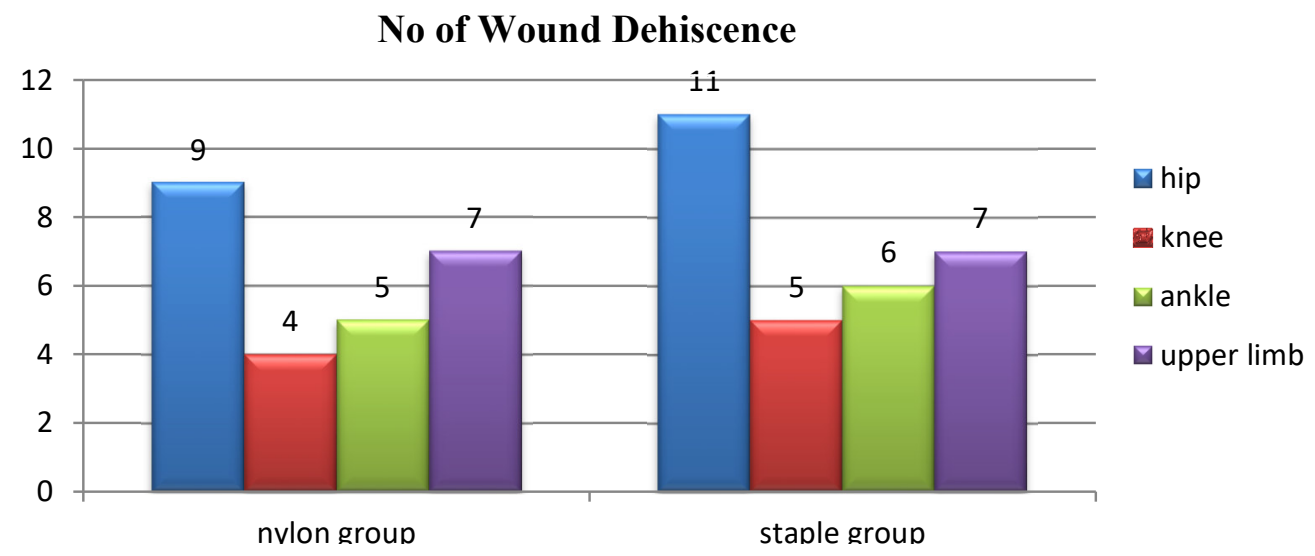

\section{Pain during Removal of Nylon/Staple}

1. Nylon group- The mean pain VAS was 1.57. Maximum patients i.e. 137 (52.29\%) had VAS score 2.

2. Staple group- The mean pain VAS score was 1.41 . Maximum patients i.e. $149(61.8 \%)$ had VAS score 1.

Secondary Procedures - Wound debridement and resuturing were needed in $4(1.65 \%)$ wounds in staple group While in suture group only $1(0.4 \%)$ wound of upper limb needed debridement and reclosure.

\section{Follow Up (6 Weeks)}

Cosmesis evaluation was done using Hollander wound evaluation score (HWES). There was no significant difference in the two study groups. The minimum HWES score was 3 and maximum was 5 in all anatomical sites. The mean HWES score in Nylon group was 4.11 and in Staple group was 4.35 .

\section{Patient Satisfaction Visual Analogue Scale}

The minimum patient satisfaction VAS score was 6 and maximum was 9 in overall orthopaedic procedures. The mean score in nylon group was 8 whereas it was 8.35 in staple group.

\section{Discussion}

Wound closure material is an afterthought for majority of orthopaedic surgeons. The combined results of several comparative trials suggest that the choice of

wound closure materials may have an impact on the rate of surgical site infections. However, the strength of the evidence is poor given the heterogeneity of the methods employed in previous studies. The current study aimed to guide the surgeons to choose closure material by determining if there is a difference in complication rates in sutured and stapled wounds.
The mean age of patients in orthopaedic procedures was 41.6 years with maximum incidence was in 20-39 year age group. It is due to high mobility of individuals in this particular age group. R.J.K. Khan et. al [6] found in their series of hip surgery $51.5 \%$ male in suture group and $55.6 \%$ male in staple group. In the current study it was observed that the in overall procedures, 348 (69.1\%) were male and $155(30.9 \%)$ were female. This wide disparity between male and female patient is because of the fact that males are exposed to wide range of trauma due to their more outdoor activities whereas females are usually domestic worker. 
Research Article

\section{Duration of skin closure}

Meiring et al [7] have recorded that there was $80 \%$ time saving with the stapling device. Ranaboldo et al [2] have found that the rate of wound closure was 8 seconds $/ \mathrm{cm}$ with stapler and 12.7 seconds/cm with sutures. Dos Santos LR et.al [8] concluded that the use of skin staples speeds up closure time by $80 \%$. Kanagaye et.al [9] observed that staplers were six times faster than standard sutures. Khan et. al [6] reported that wound closure was significantly faster with staples than with sutures $(\mathrm{P}<0.05)$. The present study showed that stapler is faster than sutures with mean duration of closure by Staples were 0.96 minutes and by Nylon it was 4.24 minutes that is 4.42 times faster than nylon group which is in concordance with above mentioned studies.

\section{Length of Incision}

Ian Stockley [10] included in his study wounds with incision size more than $18 \mathrm{cms}$ in hip and knee surgeries. R J K Khan et.al [6] observed in their study with patients undergoing total hip replacement with median incision size was $10 \mathrm{cms}$ ( 5 to $25 \mathrm{cms}$ ) in suture group and $10.5 \mathrm{cms}$ ( 7.5 to $22 \mathrm{cms}$ ) in staple group, patient undergoing total knee replacement the mean length of incision was 20 (9.0 to 25.0) in staple group and it was 19 (10.5 to 24$)$ in suture group. Present study included incision size more than $5 \mathrm{cms}$ and grouped into two groups, $<10 \mathrm{cms}$ and $>10 \mathrm{cms}$. As incision size increases there is more exposure to outer environment so there is more chances of post-operative wound complications.

\section{Wound discharge}

Bijayendra Singh et.al [11] in their study have observed there was significantly greater amount of wound discharge $(\mathrm{p}<0.009)$ in group A (clip) as compared to group B (vicryl) in hip surgery. In a recent metaanalysis conducted by Toby O Smith et.al [12] It was found that there was no significant difference in incidence of wound discharge between two methods of skin closure in hip surgery $(\mathrm{p}>0.05)$. In our study we noticed that the wound discharge was maximum at $2^{\text {nd }}$ post-op day and that the wound discharge decreases in subsequent post operative days unless wound complications occur.

\section{Infection}

Shetty AA et al [13] found that there was no superficial wound infections in vicryl suture group whereas 5 wound infections in staple group in hip surgery. Bijayendra Singh et.al [11] observed 3 cases of wound infection in stapled wound in hip surgery. Meta- analysis conducted by Toby O Smith et.al [12] found that the risk of wound infection was four times greater in those cases where the wounds were closed with staples than with sutures. In the present study, in hip surgery we observed $5(5.1 \%)$ infection in nylon group and $13(13.8 \%)$ in staple group $(\chi 2=4.3)$ and $(p=0.038)$ which was statistically significant.

Two studies studied the outcomes after use of sutures and staples in knee surgery. R J K Khan et. al [6] found 1 early wound infection out of 27 wounds in staple group and lout of 31 wounds in suture group. Late wounds infection was 3 in staple group as compared to only 1 in suture group. Ian Stockley[10] reported 1 infection out of 10 wounds in staple group and no infection was found in suture group further supporting our findings. In present study it was found that $3(6 \%)$ infection was in nylon group $8(16.6 \%)$ infection was in staple group $\chi^{2}$ was 2.8 and $(\mathrm{p}=0.09)$ that is not very much significant. Justin $\mathrm{T}$ Newman et al [14].reported that in the soft tissues surrounding the knee, particularly the area overlying the proximal tibia and patella, there may be a greater degree of vascular compromise based on wound closure technique.

Although we could not find any study those describing wound infection following different methods of skin closure after ankle surgery we observed $4(7.6 \%)$ wound infection in nylon group and $7(21.7 \%)$ in staple group with $\left(\chi^{2}=3.93\right)$ and a statistically significant $p$ value $(\mathrm{p}=0.04)$.

In one study done by Martin Murphy et.al [15] outcome of wounds closed with suture and staples after surgery in the forearm, olecranon and humerus were studied. But results were not presented independently from those for lower limb surgery. It was therefore not possible to determine whether there was a difference in outcome between these two methods of skin closure in upper limb surgery. However we observed that only $2(3.2 \%)$ wound infection were in nylon group and $5(9.4 \%)$ in staple group with $\left(\chi^{2}=1.93\right)$ and $(\mathrm{p}=0.16)$ that was not statistically significant.

Overall In the current study showed infection rate in nylon group was $14(5.3 \%)$ and $36(14.6 \%)$ in staple group. so the risk of a wound infection was over 2.8 times greater with staples than with sutures $(\mathrm{p}=0.002)$.

\section{Wound Dehiscence}

Shetty AA et. al [13] recorded in their study only one wound dehiscence with closure with staples and no wound dehiscence was found in suture group in hip 
Research Article

surgery. Toby O Smith et.al [12] in their met-analysis found no statistically significant difference between incidence of wound dehiscence between the two methods of skin closure in hip surgery $(p>0.05)$. In present study $25(9.5 \%)$ wound dehiscence found in nylon group and $29(12 \%)$ in staple group with $(\mathrm{p}=0.1)$ which is also not statistically significant.

\section{Pain during Removal of Nylon/Staple}

Shetty AA et. al[13] and Ian Stockley [10] have observed staples were more painful to remove. In the current study it was found that mean pain VAS score was 1.57 in nylon group as compared to 1.41 in staple group. Martin Murphy et.al [15] in their study observed that clip removal was found to be less painful $(p=0.001)$ thus supporting our observations.

\section{Secondary Procedure Required}

Shetty AA et al [13] reported in their study that one patient in staple group required wound debridement and secondary closure using subcuticular vicryl suture for wound dehiscence. Justin $\mathrm{T}$ Newman et.al[14] have noticed in their study that $4(4.9 \%)$ patients in suture group required a repeat operation for debridement and re-closure, one was attributed to gout and 3 were secondary to infection. In the current study it was found that wound debridement and resuturing required in 4 $(1.6 \%)$ wounds in staples group among these 2 in hip surgery, 1 each in knee and ankle surgery. While in suture group $1(0.38 \%)$ wound of upper limb was required for debridement and reclosure.

\section{Follow Up}

\section{- Cosmesis evaluation}

Martin Murphy et.al [15] observed no significant difference in cosmesis between clip and suture group at follow up. Clayer and Southwood [16] reported that the scars produced by suture closure were significantly thinner than with staples after hip surgery $(\mathrm{P}<0.05)$. Khan et al [6] in their study found (at 6 weeks follow up) HWES score[4] was 5.3 in staple group and 6 in suture group in patient who underwent total hip replacement. In present study it was found that the median HWES score (at 6 weeks follow-up) in all orthopedic surgeries was 4.11 in nylon group and 4.35 in staple group.

\section{- Patient satisfaction}

RJK Khan et al [6] reported that the mean patient satisfaction according to VAS [5] was 9.6 in staple group and 9.4 in suture group in patient underwent THR $\& 9.4$ in staple group and 9 in suture group in patients underwent TKR. In the current study it was found that there is no significant difference between two groups with means VAS scores was 8.47 in nylon group and 8.35 in staple group.

\section{Conclusion}

Hence based on present study it is concluded that the only advantage of staples is rapid skin closure yet the time saved is merely 3 to 4 minutes as compared to skin closed by suture during surgery.

A significantly higher incidence of wound infection is seen following skin closure by metallic staples in orthopaedic surgeries as compared to monofilament nylon that can be detrimental to patient safety. Traditional method of skin closure by monofilament nylon is preferable method of skin closure in orthopaedic surgery.

Funding: Nil,

Permission from IRB: Yes

Conflicts of interest: The authors report no conflicts of interest.

\section{References}

1. Mackenzie D; The history of sutures; Med Hist. Apr 1973; 17(2): 158-168;

2. Ranaboldo CJ, Rowe-Jones DC; Closure of Iaparotomy wounds: skin staples versus sutures ; Br J Surg. $1992 ; 79(11): 1172-3$.

3. Slade Shantz JA, Vernon J, Morshed S, Leiter J, Stranges G. Sutures versus staples for wound closure in orthopaedic surgery: a pilot randomized controlled trial.; Patient Saf Surg. 2013 7(1):6. doi: 10.1186/1754-94937-6

4. Hollander JE, Singer AJ, Valetine S, Henry MC. Wound registry: Development and validation. Ann Emerg Med. 1995;25:675-85.

5. Visual Analog Scale and Verbal Pain Intensity Scale: From Pain, Management: Theory and Practice, edited by RK Portenoy \& RM Tanner, copyright 1996 by Oxford University Press, Inc.

6. R. J. K. Khan, D. Fick, F. Yao,,K. Tang,,M. Hurworth,,B. Nivbrant,,D.Wood,MD, A comparison of three methods of wound closure following arthroplasty a prospective, randomized, controlled trial 10.1302/0301620X.88B2.16923 J Bone Joint Surgery 2006 ;88-B :238-242. 
Research Article

7. Meiring L, Cilliers K, Barry R, Nel CJ; A comparison of a disposable skin stapler and nylon sutures for wound closure S Afr Med J. 1982 ; 62(11):371-2

8. Santos LR dos, Freitas CA, Hojaij FC, AraújoFilho VJ, Cernea CR, Brandão LG, Ferraz AR; Prospective study using skin staplers in head and neck surgery; Am J Surg. 1995 ;170(5):451-2.

9. Kanegaye JT, Vance CW, Chan L, Schonfeld N; Comparison of skin stapling devices and standard sutures for pediatric scalp lacerations: a randomized study of cost and time benefits.; J Pediatr. 1997 ;130(5):808-13

10. Stockley and R.A. elson; Skin closure using staples and nylon sutures: a comparison of results. . Ann R CollSurg Engl. 1987; 69(2): 76-8

11. Bijayendra Singh,M. A. S. Mowbray,G. Nunn, S. Mearns; Closure of hip wound, clips or subcuticular sutures: does it make a difference? European Journal of Orthopaedic Surgery \& Traumatology 2006; 16(2):1249.

12. Toby O Smith, Debbie Sexton, Charles Mann, Simon Donell; Sutures versus staples for skin closure in orthopaedic surgery: meta-analysis; BMJ 2010; 340 ;c1199

13. Shetty AA, Kumar VS, Morgan-Hough C, Georgeu GA, James KD, Nicholl JE; Comparing wound complication rates following closure of hip wounds with metallic skin staples or subcuticular vicryl suture: a prospective randomized trial; J OrthopSurg (Hong Kong).2004 Dec;12(2):191-3

14. Justin T Newman, Steven J Morgan, Gustavo V Resende,Allison E Williams, E Mark Hammerberg,and Michael R Dayton; Modality of wound closure after total knee replacement: are staples as safe as sutures? A retrospective study of 181 patients ;PatientSaf Surg. 2011; 5: 26

15. Martin Murphy,Peter Prendergast, John Rice; Comparison of clips versus sutures in orthopaedic wound closure; European Journal of Orthopaedic Surgery \& Traumatology 2004; 14(1): 16-18.

16. Clayer M, Southwood RT; Comparative study of skin closure in hip surgery; Aust N Z J Surg. 1991 May;61(5):363-5

\section{How to cite this article?}

Gohiya A, Gupta DC, Gaur S. Comparative Study of Outcome of two Methods (Sutures Vs Staples) of Skin Closure in Orthopedic Surgery. Int J Med Res Rev 2015;3(1):16-22. doi:10.17511/ijmrr.2015.i1.04 